\title{
Evidence against hyperspecificity in implicit invariant learning
}

\author{
Ben R. Newell \\ University College, London, UK \\ James E.H. Bright \\ University of Nem South Wales, Sydney, Australia
}

\begin{abstract}
Four experiments examined the claim that cross-format transfer in invariant learning is reliant solely on the presence of repetition structure in study and test strings (Stadler, Warren, \& Lesch, 2000). Experiments 1, 2, and 3 used strings with no repetitions and found significant cross-format transfer in combination with a non-significant transfer decrement-no significant difference between same- and changed-format conditions. Further investigation of the basis of the role of repetition structure revealed an emphasis on the perceptual salience of test stimuli (Experiment 4). Our results contrast with those of Stadler et al. and suggest that under the conditions we employed invariant learning is not highly sensitive to changes in the perceptual characteristics of stimuli and therefore is inaccurately described as hyperspecific. We suggest that the term hyperspecific be reserved for cases in which minor format changes result in significant performance impairments - for example, typographical effects in implicit memory.
\end{abstract}

Despite implicit learning and implicit memory sharing the descriptor "implicit" this does not necessarily mean that they rely on the same underlying mechanism or exhibit the same characteristics. A notable difference is that implicit learning is shown to generalize to non-exposed items, whereas implicit memory reveals itself through the facilitated processing of specific, previously exposed items (e.g., Reber, 1989; Tulving \& Schacter, 1990). This paper presents experiments that investigate the degree to which one form of implicit learning (invariant learning) generalizes to novel stimuli and what the pattern of generalization elucidates about the relationship between implicit learning and implicit memory.

We might expect a mechanism that permits generalization to novel items to be less sensitive to changes in perceptual characteristics than one that requires exact matches with "old" items (Dienes \& Altmann, 1997). That is, implicit learning should be fairly robust in the face of

Requests for reprints should be sent to Ben R. Newell, Department of Psychology, University College London, Gower Street, London, WC1E 6BT, UK. Email: b.newell@ucl.ac.uk

Ben Newell was supported by a Commonwealth Scholarship. James Bright was supported by an Australian Research Council Small Grant. We would like to thank Peter Lovibond, Axel Cleeremans, Michael Stadler, and Luis Jiménez for commenting on an earlier draft of this paper. We also thank Michael Stadler for supplying the materials for Experiment 3 .

(C) 2002 The Experimental Psychology Society

http://www.tandf.co.uk/journals/pp/02724987.html

DOI:10.1080/0272498024400002 
manipulations of perceptual information, whereas implicit memory should be detrimentally affected. Implicit memory research has largely confirmed this prediction. Roediger and Blaxton (1987) demonstrated a linear reduction in priming in a word fragment completion task as the difference between the surface representation of study and test stimuli increased. The largest degree of priming resulted from visual presentation at both study and test, less from an auditory presentation of study words, and least from pictorial presentations at study. Weldon (1991) found a similar pattern of results, though failed to find any evidence of priming in the pictorial study stimuli condition. Numerous other investigations have provided further evidence that a change in the surface representation (physical and/or modal) across study and test stimuli results in the reduction or elimination of priming (e.g., Bassili, Smith, \& MacLeod, 1989; Jacoby \& Dallas, 1981; Srinivas, 1993).

With regard to implicit learning, the evidence is not so clear-cut. The prediction that implicit-learning performance should be insensitive to perceptual or surface characteristics appears to hold for some tasks but not for others. Willingham, Nissen, and Bullemer (1989) reported that after learning a repeating spatial sequence on a serial reaction time task, a change in the perceptual characteristics of the display (colours) led to the elimination of learning. Similarly, Berry and Broadbent (1988) found using a computer-simulated-control task that subjects were only able to transfer knowledge between two control tasks that exhibited the same surface characteristics (i.e., both person interaction tasks or both transport tasks). These findings suggest a relative inflexibility of the knowledge underlying implicit learning-a sentiment echoed in some reviews of the literature (e.g., Berry \& Dienes, 1991; Dienes \& Berry, 1997).

In contrast, Reber (1969) showed that subjects who memorized strings of letters generated by a finite-state grammar demonstrated savings in memorizing new strings generated by the same grammar, even when the new strings were constructed using a different letter set. This finding suggests that subjects were able to transfer some knowledge of the grammar that was not tied to the specific surface features (the letters) of the original study strings. This finding of transfer across letter sets has been replicated and extended by a number of researchers (Brooks \& Vokey, 1991; Gomez, 1997; Mathews et al., 1989; Newell \& Bright, 2001; Whittlesea \& Wright, 1997). In a similar vein Altmann, Dienes, and Goode (1995) and Manza and Reber (1997) have demonstrated transfer of knowledge across both different domains and modalities. In the Altmann et al. study subjects who studied letter strings generated by a grammar were able in a subsequent test to discriminate between sequences of tones generated by the same grammar from those generated at random. Manza and Reber found that subjects were able to transfer knowledge between sequences of tones and sequences of lights.

Transfer effects consistent with those found in the artificial-grammar tasks have also been observed with the invariant-learning procedure of Burton and colleagues (e.g., Bright \& Burton, 1994, 1998; McGeorge \& Burton, 1990; Wright \& Burton, 1995). Bright and Burton (1994, 1998) induced a preference for schematic clocks bearing a time between 6 and 12 o'clock, which transferred from analogue to digital representations of time (and vice versa). They found in fact, consistently better performance when the format changed from analogue at study to digital at test, than when both study and test times were in analogue format. Furthermore, in the original demonstration of invariant learning, McGeorge and Burton found that an induced preference for number strings containing an invariant digit persisted when study strings were presented as digits (e.g., 1234) and test strings as their word equivalents (e.g., one 
two three four). Thus, the evidence from the invariant- and artificial-grammar learning paradigms suggests that the knowledge underlying performance is flexible in that it can be transferred between perceptually different domains (Dienes \& Altmann, 1997).

The contrast in the flexibility of knowledge in different implicit-learning tasks may be attributable to the nature of experimental instructions used in the tasks. Tasks that instruct participants to use knowledge that they have been exposed to during training (e.g., artificialgrammar learning, invariant learning), are less sensitive to changes in perceptual characteristics than those in which no instruction is given (e.g., serial reaction time tasks, control tasks). Performance on implicit-memory tasks, similarly, is presumably a reflection of nonintentional or non-instructed retrieval (Schacter, Bowers, \& Booker, 1989) and hence shows sensitivity to changes in perceptual characteristics. This, of course, is not such an astonishing hypothesis: Given an explicit instruction as to the relevance of previously exposed information to the task at hand, participants are able to use this information more efficiently than when not instructed. This notion of participants actively adapting information to suit the particular demands of tests of implicit learning has been most clearly formulated by Whittlesea and colleagues (Whittlesea \& Dorken, 1993; Whittlesea \& Wright, 1997).

A question of considerable theoretical interest, then, concerns the aspects of information that participants utilize on transfer tests of implicit learning. The finding of a transfer decrement - the reduction in performance between transfer and same-format/domain performance-in many of the grammar-learning studies suggests that whatever information supports learning some of it must be bound to the specific perceptual features of the stimulus materials (Dienes \& Altmann, 1997). By the same token, the fact that some significant transfer is still observed means that at least some of the information is flexible and free of the specific materials upon which it is instantiated in the study phase.

In the implicit-memory literature the term hyperspecificity has been used to describe the nature of the information underlying the phenomenon. The prefix hyper implies, in our minds, an excessive sensitivity to any change in format or modality. As such, it is an appropriate term to describe the information supporting implicit memory, because changes as trivial as the font in which study and test items are written can have a detrimental effect on performance (Jacoby \& Hayman, 1987). In contrast, this notion of an excessive sensitivity does not sit well as a description of implicit learning - at least, not in those tasks in which significant transfer performance is observed. Part of the problem in using the term hyperspecificity is in deciding what should be the null hypothesis: no learning or no transfer decrement? If the latter is accepted then we are left with the problem of deciding how much decrement implies how much specificity - should a decrement of $10 \%$ but intact (i.e., significant) transfer imply that the knowledge is hyperspecific and a decrement of $50 \%$ combined with no transfer indicate hyperhyperspecific knowledge? This point demonstrates the ill-defined nature of the term hyperspecificity and therefore its inadequacy as a description of the information supporting learning. To be sure, findings of transfer decrement indicate that the information underlying learning is tied to some extent to perceptual features, but if transfer is still significant then it seems inappropriate to label this information as excessively sensitive to changes in format.

To explore the issue of hyperspecificity and the adequacy of its use as a description of implicit learning, we examined recent claims of Stadler, Warren, and Lesch (2000) that invariant learning - one of the tasks that we expect to be relatively insensitive to changes in perceptual characteristics-is hyperspecific. 
In the Stadler et al. (2000) procedure, subjects are shown 40 four-digit number strings in an incidental-learning task (taken from McGeorge \& Burton, 1990). All the strings presented to the subjects contain the digit 3 , though this invariant feature is not brought to the attention of the subjects. In a subsequent surprise test phase, subjects are shown 20 pairs of novel fourdigit strings where one string in each pair contains a 3 (the positive) and the other does not (the negative). Subjects are told (falsely) that they have seen one and only one of the strings in the previous study phase, and they are instructed to choose the one they think they have seen before. The reliable finding is that subjects choose more positives than would be expected by chance (typically $60 \%$ or $70 \%$ ). As noted earlier, McGeorge and Burton found that this preference persisted when the surface representation of strings was changed from digits at study to their word equivalents at test.

One explanation of this cross-format transfer was provided by Cock, Berry, and Gaffan (1994), who demonstrated that subjects were more likely to select a string that does not follow the invariant rule but is similar to one of the study strings than one that does follow the rule but is not similar to one of the study strings. They went on to speculate that similarity information of this nature could provide the basis for "some form of mental translation" in which subjects "pictured" test strings in their original form, or be based on abstract or relational similarity in which exemplars are stored in a non-hyperspecific form (p. 1031). They did not, however, test this similarity explanation of cross-form transfer.

Stadler et al. (2000), however, drew on a different study by Wright and Burton (1995) to explain the cross-format transfer effect. Wright and Burton discovered that the presence or absence of the digit 3 in the number strings was correlated with the probability of repeated digits appearing in the strings. Specifically, they showed that negative strings are more likely than positive strings to contain repeated digits. Repeated digits in a string are thought to make it distinctive (e.g., 5662 or 4784), thus enabling subjects to reject the string as an item that they think they have not seen in the study phase of the experiment. Due to the correlation between these two sources of information (invariant 3 and repeated digits) it may appear that subjects make their selections on the basis of (implicit) knowledge of the invariant 3 . However, the results suggest that subjects are relying on negative knowledge about the relative absence of repeated digits in the study phase strings. By manipulating the presence and absence of repetitions in a specifically designed test set, Wright and Burton demonstrated that subjects' selections were predominantly mediated by a "reject distinctive items" strategy.

Stadler et al. (2000) argued that the cross-form transfer (digits to their word equivalents) observed in the original McGeorge and Burton (1990) task was also mediated by the rejection strategy. They demonstrated in two experiments that in conditions where neither study nor test items contained repetitions (rendering the rejection strategy ineffective) learning in the cross-form transfer condition disappeared. However, when repetitions were added to test items (Experiment 3), learning in the cross-form transfer condition returned. Stadler et al. interpreted these results as evidence for hyperspecificity of learning in the invariant task. This is because learning is only observed in conditions where the specific physical form of study and test items is the same. In conditions where format is changed between study and test, and, crucially, subjects cannot rely on the rejection strategy, learning is absent.

Under such an interpretation the only basis of learning that can transfer across surface formats is the rejection strategy. Our own work confirms that the rejection strategy does transfer (Newell \& Bright, 2002) but we suggest that the claim that this is the only transferable basis of 
learning is too strong. First, if one accepts the argument that finding a transfer decrement is not the same as saying that learning is hyperspecific, then if any significant transfer is found, some other bases of learning must be responsible for this performance. Both Stadler et al.'s own data and data recently collected in our laboratory (Newell, 2000) point to the suggestion that some learning remains, and therefore some information other than that embodied in the repetition structure of strings must be transferable. Second, the overwhelming evidence from other demonstrations of implicit learning in which instructions as to the relevance of study phase information are given suggest that a portion of this information is flexible enough to be transferred and therefore inaccurately characterized as hyperspecific.

\section{EXPERIMENT 1}

Experiment 1 compared the invariant selection performance of subjects given digit strings (e.g., 1234) at both study and test with those given digit strings at study and word strings (e.g., one two three four) at test. Consistent with Stadler et al. (2000, Experiments 1 and 2), no repeated digits appeared anywhere in the study or test strings. This manipulation ensured that subjects could not rely on the rejection strategy. Subjects engaged in one of two orienting tasks. The arithmetic task (used in the Stadler et al. study) required subjects to compare the totals of the first two and second two digits in the four-digit strings. The other orienting task (taken from McGeorge \& Burton, 1990) required subjects to count the number of straight lines in each four-digit string. The task was included because, as McGeorge and Burton (see also Berry \& Dienes, 1991) pointed out, cross-form transfer might operate through the maintenance of the same phonological code between study and test strings (i.e., "1" and "one" have the same phonological code). It is suggested that subjects engaged in the more perceptualbased line-counting task are less likely (than subjects engaged in the arithmetic task) to process the digits as semantic units and are therefore less likely to encode the digits phonologically (McGeorge \& Burton, 1990). This should act to mitigate any transfer between different surface representations. If the rejection strategy is the only transferable basis of learning in the invariant task we expected to find no learning in the cross-format conditions.

\section{Method}

\section{Subjects}

A total of 150 students in introductory psychology classes at the University of New South Wales participated in the experiment to fulfil course requirements. All subjects saw strings as digits during the study phase. Of those subjects who performed the line-counting task during study, 36 saw strings as digits at test, and 41 saw strings as words. Of the subjects who performed the arithmetic task, 33 saw strings as digits at test, and 40 saw strings as words. Subjects were tested in groups with all subjects in a group performing the same orienting task. None of the subjects had participated in any previous research concerning implicit learning.

\section{String construction}

Two databases were created from the list of all four digit numbers in which no digits were repeated. The first database contained numbers with the digit 3 (positive strings); the second database contained numbers without the digit 3 (negative strings). A total of 30 strings were selected from the first 
database - these served as the study items. To guard against the possibility that subjects might rely on episodic knowledge of the answers elicited from the arithmetic orienting task (Churchill \& Gilmore, 1998), the number of strings in which the sum of the first two digits was greater than, less than, or equal to the sum of the second two digits was equated across the 30 study strings. A further 18 items were selected from the first database and were paired with 18 items from the second database. The resulting 18 pairs of positive and negative strings served as the test pairs. In addition, three strings $(9835,2167,5674)$ were chosen for instructing students in how to perform their respective orienting tasks. For the arithmetic groups, the strings provided examples of when the sum of the first two digits was greater than, less than, or equal to the sum of the second two digits. For the line-counting groups, the examples included all individual digits from 1 to 9 , enabling subjects to count the number of straight lines in each possible digit. The stimuli were printed on overhead projector slides with approximately 10 stimuli on each slide. During the study and test phases stimuli were presented individually by using a mask to cover the other stimuli on the slide. The left/right positioning of positives and negatives in the test pairs was counterbalanced. All study and test strings are listed in the Appendix.

\section{Procedure}

Subjects were seated in a classroom in groups of between approximately 15 and 25 . The slide showing the practice strings was placed on an overhead projector, and the experimenter worked through each example with the subjects. Subjects were led to believe that completing the orienting task was the purpose of the experiment-no mention was made of the subsequent test phase. Each study string was shown for approximately $5 \mathrm{~s}$ and subjects recorded their responses for each string on an answer sheet provided.

On completion of the study phase, subjects were told that their memory for the previously presented numbers was to be tested. They were told that they had seen one number in each of the to-be-presented pairs and that they had not seen the other number. Subjects were required to indicate on their answer sheet whether the string on the left or the right was the one they had seen before. They were asked not to leave any answers blank and to guess if they were unsure. The 18 test pairs were then presented one at a time for approximately $5 \mathrm{~s}$.

\section{Results and discussion}

The upper section of Table 1 shows the mean selection of strings following the invariance rule from the four conditions of the experiment.

A 2 (orienting task: line counting, arithmetic) $\times 2$ (test format: digit, word) betweensubjects analysis of variance revealed a main effect of orienting task, $F(1,146)=14.40, p<$ .001 , no main effect of test format, $F(1,146)=1.54, p>.20$, and no interaction, $F(1,146)=$ $.220, p>.50$. Subjects who performed the line-counting task selected strings following the invariance rule at rates significantly different from chance performance $(50 \%)$ in both sameformat and changed-format conditions: $t(35)=2.56, p<.05 ; t(40)=3.00, p<.01$, respectively. A stronger invariant selection effect was found for subjects who engaged in the arithmetic task. Rates of selection differed significantly from chance performance $(50 \%)$ in both same- and changed-format conditions: $t(32)=7.11, p<.001 ; t(39)=5.71, p<.001$.

Learning was observed in the cross-form transfer condition even though study and test items contained no repeated digits. This finding demonstrates that cross-form transfer can occur when the rejection strategy is rendered ineffective and that therefore the rejection strategy is not the only transferable basis of learning. The finding implies that subjects are 
TABLE 1

Mean percentage selection of positive strings by condition in

Experiments 1 to 3

\begin{tabular}{lllll}
\hline & & & \multicolumn{2}{c}{ Test format } \\
\cline { 4 - 5 } Experiment & Orienting task & Study format & Digit & Word \\
\hline 1 & Line count & Digit & 56.3 & 54.7 \\
& Arithmetic & Digit & 65.0 & 61.5 \\
& & & & \\
& Line count & Digit & 57.0 & 55.9 \\
& Arithmetic & Digit & 67.3 & 57.9 \\
& Arithmetic & Digit & 52.7 & 59.1 \\
& Arithmetic & Word & 58.6 & 59.5 \\
\hline
\end{tabular}

using information that is not embodied in the repetition structure of the strings. Furthermore, the non-significant effect of format indicates that this information can be equally well used under both same- and changed-format conditions.

A question then arises as to what this information might be. One potential candidate is similarity information of the type identified by Cock et al. (1994). To examine this possibility an exploratory similarity analysis was conducted on a subset of subjects' data.

Similarity analysis. Four simple indices of similarity between the test strings and the learning strings were computed (the indices were taken from Cock et al., 1994). Each test string was examined, digit by digit, and compared with each of the 30 learning strings. All subjects saw the same learning and test strings so this analysis only needed to be performed once. The indices computed were: (1) total digits in common, regardless of position; (2) digits in the same position within the two strings; (3) adjacent pairs of digits in common regardless of position; and (4) adjacent pairs of digits in the same position. Indices 3 and 4 were included because for the arithmetic task subjects were required to sum adjacent digits, so they may have developed a sensitivity to these pairings. One point was awarded for every digit that satisfied a particular index (e.g., 3689 compared with 1328 would have a score of 2 - digits 3 and 8 in common but not in the same position —whereas 1593 and 1523 would have a score of $5-1,5,3$ in common, 15 an adjacent pair and in the same position).

A total of 10 subjects were selected at random from each of the four conditions of the experiment for the similarity analysis. As this was only an exploratory analysis, it was decided that a sample size similar to the one used by Cock et al. (1994, 12 subjects) would be sufficient to demonstrate any similarity effects that might be present. Following Cock et al., the four indices described earlier were summed separately for each test string across the 30 learning strings for 10 subjects from each condition. The mean score was then calculated for four categories of test string within the individual subject data: positive strings that were selected, positives that were rejected, negatives selected, and negatives rejected. The numbers of rejected positive and selected negative strings were lower than those of the other two categories, making their means less reliable, however, all subjects did select some negative strings (range 3-14 out of a possible 18). 
Table 2 displays the means across 10 subjects from the arithmetic orienting task/same format condition of Experiment 1, for the four categories of test string. The means are grouped for test pairs in which the positive was selected over the negative and for pairs in which the opposite selection occurred.

Table 2 shows that positive strings are more similar than negative strings across all four indices of similarity. This is unsurprising as every positive string contained the invariant digit 3 and therefore had at least one digit in common with every learning string. There appears to be no evidence for an effect of similarity operating independently of the presence of the invariant digit. Selected negative strings were no more similar than rejected negative strings on any of the indices of similarity measured (all $p \mathrm{~s}>.1$ ). Moreover, there were no significant differences in the similarity scores of selected and rejected positive strings. If subjects were relying on specific item similarity, selected negative and positive strings should exhibit significantly higher similarity scores than their rejected counterparts.

It appears that specific item similarity did not play a role in determining performance in the arithmetic same condition, at least for this subset of 10 subjects. A parallel analysis revealed no independent effects of similarity in the arithmetic orienting task/changed-format condition either. Table 3 displays the means for this condition.

It should be noted that the subsets of subjects used in these similarity analyses did perform above chance in terms of selecting strings conforming to the invariant rule. The mean for the subjects from the same-format condition was $64.4 \%$, significantly above chance performance of $50 \%, t(9)=4.33$; for the changed group it was $64.5 \%$, also significantly above chance, $t(9)=$ 3.56. Thus the failure to find a similarity effect is unlikely to be due to the subset of subjects selected not paying sufficient attention to the task. Comparisons of selected and rejected negative and positive strings in the line-counting conditions failed to reveal any evidence for an independent effect of similarity.

The failure to find a similarity effect in the arithmetic same condition is in direct contrast to the findings of Cock et al. (1994). They report that selected negatives were significantly more similar than rejected negatives both in terms of total adjacent pairs and in terms of the total number of digits in common. The reason for this discrepancy may be the exclusion of repeated digits in the strings used in Experiment 1. Including repeated digits in both learning and test strings, as Cock et al. did, may have led to a higher proportion of digits in common and thus an increased source of similarity information.

TABLE 2

Experiment 1: Mean similarity scores for four categories of test string (arithmetic/same format condition)

\begin{tabular}{lccccc}
\hline & \multicolumn{2}{c}{ Positive selected } & & \multicolumn{2}{c}{ Negative selected } \\
\cline { 2 - 3 } \cline { 6 - 7 } Similarity index & Positive & Negative & & Positive & Negative \\
\hline 1. Total digits in common & 62.62 & 44.92 & & 63.51 & 44.97 \\
2. Total digits in same position & 14.47 & 10.12 & & 14.9 & 10.71 \\
3. Adjacent pairs in common & 5.72 & 2.49 & & 5.77 & 2.5 \\
4. Adjacent pairs in same position & 2.24 & 1.02 & & 2.43 & 1.12 \\
\hline
\end{tabular}


TABLE 3

Experiment 1: Mean similarity scores for four categories of test string (arithmetic/changed format condition)

\begin{tabular}{|c|c|c|c|c|}
\hline \multirow[b]{2}{*}{ Similarity index } & \multicolumn{2}{|c|}{ Positive selected } & \multicolumn{2}{|c|}{ Negative selected } \\
\hline & Positive & Negative & Positive & Negative \\
\hline 1. Total digits in common & 62.55 & 45.17 & 63.65 & 44.5 \\
\hline 2. Total digits in same position & 14.27 & 10.34 & 15.6 & 10.68 \\
\hline 3. Adjacent pairs in common & 5.94 & 2.36 & 5.95 & 2.5 \\
\hline 4. Adjacent pairs in same position & 2.33 & 1.01 & 2.51 & 0.95 \\
\hline
\end{tabular}

The results of Experiment 1 suggest that cross-format transfer in the invariant-digit task is not based on knowledge of repetitions, nor is it based on "mental translation" or abstract or relational similarity information. This leads us to suspect that information about the invariant digit itself is transferable across surface formats. However, before drawing any strong conclusions a few issues need addressing.

It has been suggested that a common phonological code between study and test constitutes a further possible basis for transfer (Berry \& Dienes, 1991; McGeorge \& Burton, 1990). This phonological information may have been used by subjects engaged in the arithmetic task; however, it was argued that it was less likely that such information was used by subjects engaged in the more perceptual-based line-counting task.

The line-counting task was used in an attempt to suppress phonological encoding of study and test strings. It is plausible, however, that subjects still used the same code when processing study and test strings. For example, because all the strings appeared in the same font it is feasible that subjects might learn quickly that a 7 contained two straight lines, a 4 three straight lines, and so on. They could then use this truncated form of processing to add the "straight line totals" of the digits in the string together. Thus subjects might still encode study strings by first pronouncing them and then writing down the line total. If this were the case, it would act against the proposed suppression of phonological encoding. In Experiment 2, an attempt was made to reduce further the possibility that the same phonological code mediates transfer, by encouraging subjects to pronounce study and test strings with different phonological codes.

\section{EXPERIMENT 2}

Different phonological codes were used for study and test strings. At study, strings were presented as numbers, consistent with Experiment 1, but at test strings were presented as prices. This manipulation ensured that the phonological code changed from, for example, "three four two seven" at study, to "thirty-four dollars and twenty-seven cents" at test. This change in phonological code is argued to increase the difficulty in inducing a mapping between the study and test representations and thus mitigate the chances that transfer is mediated via phonological encoding. Again, if the rejection strategy is the only transferable basis of learning we expect no learning to occur in the cross-format transfer condition. 


\section{Method}

The method was the same as that of Experiment 1 with the following exceptions:

\section{Subjects}

A total of 83 new subjects from the same pool as that of Experiment 1 participated in this experiment. All subjects saw strings as digits during the study phase. Of those subjects who performed the linecounting task during study, 23 saw strings as digit representations of prices at test, and 16 saw strings as word representations of prices. Of the subjects who performed the arithmetic task, 23 saw strings as digit representations of prices at test, and 21 saw strings as word representations of prices.

\section{Materials}

The slides used in the test phase of Experiment 1 were retyped so that the digit strings contained a $\$$ sign and a "." separating the first and second digits (e.g., \$12.34). The word strings were retyped as prices (e.g., twelve dollars and thirty-four cents).

\section{Results and discussion}

The middle section of Table 1 shows the mean selection of strings that followed the invariant rule for the four conditions. The pattern of results is similar to that found in Experiment 1. A 2 (orienting task: line counting, arithmetic) $\times 2$ (test format: digit price, word price) betweensubjects analysis of variance revealed a main effect of orienting task, $F(1,79)=5.41, p<.05$; the main effect of test format just missed significance, $F(1,79)=3.92, p>.051$; the interaction was not significant, $F(1,79)=2.45, p>.10$. Comparisons to chance performance revealed that subjects selected the strings following the invariant rule at a rate significantly above chance in all four conditions: line counting, digit price, $t(22)=2.26, p<.05$; line counting, word price, $t(15)=2.25, p<.05$; arithmetic, digit price, $t(22)=10.5, p<.01$; arithmetic, word price, $t(20)$ $=2.72, p<.05$.

Experiment 2 examined whether the cross-form transfer observed in Experiment 1 was mediated through a sustained phonological code between study and test strings. In conditions where both the format and the phonological code were changed learning was observed. This learning occurred even though subjects were unable to rely on the reject repetitions strategy. The effect of format showed a strong trend towards a significant transfer decrement-poorer performance in the changed-format condition. However, the significant learning still observed in this condition demonstrates that at least some of the information supporting learning is not bound to the specific perceptual or phonological aspects of the study materials, and is therefore, we believe, inaccurately characterised as hyperspecific.

No effect of specific item similarity was found in Experiment 1, and, given that the same study and test strings were used in Experiment 2, we decided not to perform a similarity analysis on the data.

It is tempting to conclude on the basis of Experiments 1 and 2 that information pertaining to the invariant digit is transferable across surface formats. However, we are aware that there is a range of features in a list of 30 four digit numbers that might be correlated with the presence of the invariant. To guard against the possibility that our findings reflected idiosyncracies in our materials, Experiment 3 attempted a replication using the set of strings employed by 
Stadler et al. (2000). Experiment 3 also sought to replicate Stadler et al. more closely to ensure that methodological differences were not responsible for our discrepant findings.

\section{EXPERIMENT 3}

The study and test strings used by Stadler et al. (2000) were used, and a full-factorial design that varied formats at both study and test (as used by Stadler et al.) was employed. Such a design is preferable because measurement of invariant selection occurs under exactly the same conditions for both transfer and non-transfer groups.

\section{Method}

\section{Subjects}

A total of 44 students from the same subject pool as that of Experiment 1 participated in the experiment to fulfil course requirements. All subjects performed the arithmetic orienting task. Subjects were allocated at random to one of the four conditions resulting from the 2 (study format: digits, words) $\times 2$ (test format: digits, words) design. There were equal numbers of subjects in each condition, and each subject was tested individually.

\section{Materials}

The digit sets used by Stadler et al. (2000) were obtained. These consisted of a training list of 40 fourdigit numbers all containing the digit 3 and a test list of 20 pairs of four-digit numbers with one number in each pair containing a 3 . Repeated digits did not appear in either list. Lists were printed in booklets with each training item or test pair appearing on a separate page. The order of presentation of training items and test pairs was the same as that used by Stadler et al. All the strings are listed in the Appendix.

\section{Procedure}

The procedure replicated Experiments 1 and 2, with the exception that testing was done on an individual basis, and subjects worked through the training and test booklets at their own pace.

\section{Results and discussion}

The lower section of Table 1 displays the mean selection of the strings that followed the invariant rule in the four conditions. A 2 (study format: digits, words) $\times 2$ (test format: digits, words) between-subjects analysis of variance revealed no main effect of study format, $F(1,40)=1.21$, $p>.2$, no main effect of test format, $F(1,40)=1.58, p>.2$, and no interaction between these factors, $F(1,40)=0.88, p>.3$. Given that this interaction was not significant, there is no suggestion that performance at test was better when study and test formats matched than when they differed. Indeed, subjects trained and tested on different stimulus formats selected the invariant-containing string $58 \%$ of the time, a rate significantly above chance $(50 \%), t(21)=$ $3.93, p<.01$. Similarly, subjects trained and tested on the same stimulus format selected strings following the invariance rule on $56 \%$ of occasions, also a rate above chance, $t(21)=$ $3.30, p<.01$. There was no significant difference between these rates of selection, $t(42)=0.88$, $p>.1$. We acknowledge that the uncharacteristically low score for the digit-digit group is somewhat responsible for this pattern of results, and we are not sure why this happened. 
However, the overall pattern of results replicates those found in Experiments 1 and 2: Learning occurs under both same- and cross-format transfer conditions, and there is no evidence of a transfer decrement. Finding this pattern with two different sets of materials in both group and individual testing and when both study and test formats are manipulated suggests that the cross-format effect is attributable neither to idiosyncrasies of materials nor to particular experimental designs.

A similarity analysis revealed, consistent with Experiment 1, no evidence for an effect of similarity operating independently of the presence of the invariant digit. Tables 4 and 5 display the mean similarity scores for the four indices (taken from Cock et al., 1994) for the digitdigit and the word-word conditions, respectively. There were no significant differences between the similarity scores of selected and rejected negative and positive strings in either condition (all $p \mathrm{~s}>.1$ ). Similarity analyses were not performed on the data from the crossformat transfer conditions as it was thought unlikely that similarity would operate under cross-format conditions when no evidence for its operation had been found in the same-format conditions.

The results from the three experiments appear to provide strong evidence that information other than that embodied in the repetition structure of strings is transferable across surface formats. This information does not appear to be based on specific item similarity (at least not of the type captured by the similarity indices employed here), leading us to suspect that the information pertains to the invariant digit.

We return to the issue of whether this information is best described as hyperspecific in the General Discussion. In the final experiment we turn to the question of what role repeated digits might play in the invariant task. Although the results of Experiments 1, 2, and 3 indicate that cross-form transfer does not rely exclusively on subjects' use of the rejection strategy, Stadler et al. (2000, Experiment 3), Wright and Burton (1995), and Newell and Bright (2002) have all clearly demonstrated that when subjects are able to use the rejection strategy their selections are consonant with rejection strategy predictions. Independent of the presence or absence of the invariant 3 , subjects tend to reject the strings containing repeated digits.

The rejection effect has been interpreted in terms of the salience of strings containing repeated digits (Wright \& Burton, 1995). Presumably this salience manifests itself in the perceptual pattern of the string (i.e., the string 2448 is more salient on a perceptual basis than the string 2390). If this assertion is correct, we might expect to find that subjects who engage in an orienting task that emphasizes the perceptual properties of the strings (the line-counting task)

TABLE 4

Experiment 3: Mean similarity scores for four categories of test string (digit-digit condition)

\begin{tabular}{|c|c|c|c|c|}
\hline \multirow[b]{2}{*}{ Similarity index } & \multicolumn{2}{|c|}{ Positive selected } & \multicolumn{2}{|c|}{ Negative selected } \\
\hline & Positive & Negative & Positive & Negative \\
\hline 1. Total digits in common & 83.07 & 59.92 & 84.00 & 60.33 \\
\hline 2. Total digits in same position & 19.53 & 14.38 & 18.87 & 13.92 \\
\hline 3. Adjacent pairs in common & 2.30 & 0.87 & 2.38 & 1.03 \\
\hline 4. Adjacent pairs in same position & 1.39 & 0.60 & 1.28 & 0.59 \\
\hline
\end{tabular}


TABLE 5

Experiment 3: Mean similarity scores for four categories of test string (word-word condition)

\begin{tabular}{lcccccc}
\hline & \multicolumn{2}{c}{ Positive selected } & & \multicolumn{2}{c}{ Negative selected } \\
\cline { 2 - 3 } Similarity index & Positive & Negative & & Positive & Negative \\
\hline 1. Total digits in common & 83.66 & 60.35 & & 83.60 & 60.09 \\
2. Total digits in same position & 19.35 & 13.96 & & 19.04 & 14.29 \\
3. Adjacent pairs in common & 2.32 & 0.92 & & 2.44 & 0.98 \\
4. Adjacent pairs in same position & 1.34 & & 0.58 & & 1.39 & 0.60 \\
\hline
\end{tabular}

to show better performance than those who carry out a task that encourages non-perceptual processing (the arithmetic task). Experiment 4 examined this possibility.

\section{EXPERIMENT 4}

In Experiment 4 both study and test strings were presented to all subjects as digits. We were interested in whether the suggested perceptual salience of strings containing repeated digits would give rise to better performance for subjects engaged in the line-counting task.

\section{Method}

The method followed that of Experiment 1, with the following exceptions:

\section{Subjects}

A total of 34 new subjects from the same pool as that of the previous experiments participated. Of these, 17 subjects performed the linecounting orienting task, and 17 performed the arithmetic task.

\section{Materials}

The same 30 learning strings as those used in Experiments 1 and 2 were used. The 18 test pairs from Experiment 2 were used, with repetitions added to the negative string in 12 cases and the positive string in 6 cases. For both negative and positive strings two thirds of the strings contained contiguous doubles in positions 1 and 2, 2 and 3 , and 3 and 4 (e.g., 7742, 6112, 3422); the remaining third contained noncontiguous doubles (e.g., 2324, 5625). This distribution reflects the approximate proportion of repetitions found in the populations of positive and negative strings (Wright \& Burton, 1995). The test strings are listed in the Appendix.

\section{Results and discussion}

The mean selection of strings following the invariant rule was $67.3 \%$ for the line-counting group and $60.4 \%$ for the arithmetic group. These two means were significantly different from each other, $F(1,32)=4.96, p<.05$. The direction of the effect indicates that subjects performing the line-counting task exhibited a higher degree of learning than those who carried out the arithmetic task. Strings containing the invariant were selected at a rate significantly different from chance in both conditions $(p<.05)$. Analysis of selections with respect to rejection of 
repeated digits (independent of the invariant 3 ) revealed that subjects in the line-counting condition rejected strings containing repetitions on $85.6 \%$ of occasions compared to $76.1 \%$ of occasions for those in the arithmetic task condition. The results support the proposal that the rejection of salient strings drives subjects' performance. The "reversed levels of processing" effect (greater learning for subjects engaged in the perceptual compared to the semantic taskopposite to the pattern found in Experiments 1 and 2) suggests that it is the perceptual pattern of strings containing repeated digits that makes them salient.

\section{GENERAL DISCUSSION}

The experiments presented here examined the claim that the rejection strategy is the only basis of learning that transfers across surface formats in the invariant-learning task (Stadler et al., 2000). Experiments 1, 2, and 3 found that significant learning remained under changedformat conditions - even when the rejection strategy was rendered ineffective. Thus some other form of information that is not embodied in the repetition structure of strings must be transferable across surface formats. This information does not appear to be based on abstract or relational similarity between study and test strings (e.g., Cock et al., 1994), nor on a common phonological code across study and test strings (e.g., Berry \& Dienes, 1991; McGeorge \& Burton, 1990). Whatever the information, we contend that given a pattern of significant learning combined with non-significant transfer decrement in three experiments, describing invariant learning as excessively sensitive to changes in perceptual characteristics (i.e., hyperspecific) is inaccurate.

We suggest that the term hyperspecificity be reserved for cases in which minor changes in the format of study and test materials affect performance. For example, the finding that successive tests of implicit memory with different cues (e.g., using $\mathrm{CH}_{-} \_\mathrm{R}$ versus _HA_R as word fragments for the target CHAIR) are stochastically independent (Tulving \& Schacter, 1990). In other words, even if a subject produces the target when given one fragment, one cannot predict that they will produce the target when given the other fragment. Gardiner, Dawson, and Sutton (1989) reported comparable findings in a task in which subjects generated words from fragment clues during study (e.g., single unmarried man-B___E_OR) and then performed a fragment completion task. Subjects given the same fragment at study and test demonstrated greater priming than if they had generated the target from a different fragment during study. These effects appear to be mediated by a quite different form of information than that which underlies the more radical transfer effects observed in the current experiments (e.g., digits to word price equivalents) and other demonstrations of invariant learning (e.g., Bright \& Burton, 1994, 1998) and artificial-grammar learning (e.g., Altmann et al., 1995; Manza \& Reber, 1997). Labelling both forms of information as hyperspecific does not serve to illustrate this difference.

On the basis of our experiments we cannot unequivocally identify the information supporting learning in the invariant task. Great care was taken in the construction of the strings to avoid correlated information (e.g., repetitions, the episodic similarity of additions) but we concede that performance may have depended on unidentified features. However, consistent with previous investigations of the invariant task in which materials have been controlled in a variety of ways (Churchill \& Gilmore, 1998; Cock et al., 1994; Wright \& Burton, 1995) the data suggest a possible residual role for the invariant feature. In the current study, both repetition 
and similarity information were ruled out, and yet significant learning was still found in three experiments - it is tempting to conclude that this learning was supported by information pertaining to the number 3 .

A question that always arises in investigations of implicit learning is whether the information underlying learning was consciously or unconsciously mediated. We did not seek to answer this question directly in the current experiments; however, we did employ a simple test of awareness in all four experiments. On completion of the test phase subjects were told that all study strings conformed to a simple rule and were asked to write down what they thought that rule might be. No subject correctly identified the invariant 3 rule. However, in Experiment 4 a number of subjects noted that no repeated digits had been present in the study strings. This latter finding highlights the problems in using verbal reports as measures of awareness. The appearance of performance based on implicit knowledge may be an artefact of failing to tap subjects' conscious knowledge exhaustively (see Shanks \& St. John, 1994). By only asking one question that focused on the invariant rule we may have failed to elicit other conscious knowledge that may have been responsible for performance. Although we did not pursue this issue in the present study, evidence from other investigations suggests that invariant learning may involve conscious knowledge (Newell \& Bright, 2002; Wright \& Burton, 1995).

Finally, Experiment 4 illustrated that the flexibility with which information is deployed is a reflection of the interplay between the nature of experimental instructions and the demands of the orienting and test tasks. In Experiments 1 and 2, in which repeated digits were absent, subjects who engaged in the semantic orienting task performed significantly better than those who performed the perceptual task. This depth of processing effect presumably reflects the stronger (false) memory trace produced by encoding the digits as semantic units as opposed to perceptual patterns. Experiment 4 demonstrated a reversal of this "depth" effect, which illustrates the interplay between instructions and demands. An orienting task that encouraged subjects to process the study strings as perceptual patterns led to the increased salience of test strings containing repeated digits.

This interplay between instructions and demands is at the heart of processing accounts of implicit learning — arguably the current dominant theoretical perspective (Cleeremans, Destrebecqz, \& Boyer, 1998). The central tenet of these accounts (e.g., Whittlesea \& Dorken, 1993; Whittlesea \& Wright, 1997) is that the expression of learning depends on the degree of match between the processing operations engaged in during study and test. The more congruent study and test processing operations are, the greater the expression of learning. The experiments reported here support this processing view and highlight that it is the overlap in processing operations (in the sense that Kolers, 1973, originally used this term) and not specific formats that is of primary importance in predicting performance.

In conclusion, we contend that implicit memory and implicit learning may be distinguished in terms of the specificity of the information that mediates their effects. This difference in specificity may simply be a reflection of task demand differences. That is, in tasks in which instructions highlight the relevance of previously exposed material (e.g., invariant learning and grammar learning) information can be deployed flexibly; whereas in tasks in which no reference is made to studied material (e.g., serial reaction tasks, complex control tasks, and all implicit memory tasks) deployment of information is inflexible. Alternatively it may be a reflection of the operation of separate underlying mechanisms - one that allows generalization to novel items and another that produces sensitivity to specific matches. Further 
research that delineates the way in which different task demands and encoding and test conditions determine the specificity of underlying knowledge will help to distinguish between these two alternatives.

\section{REFERENCES}

Altmann, G.T.M., Dienes, Z., \& Goode, A. (1995). Modality independence of implicitly learned grammatical knowledge. Fournal of Experimental Psychology: Learning, Memory, and Cognition, 21, 899-912.

Bassili, J.N., Smith, M.C., \& MacLeod, C. (1989). Auditory and visual word stem completion: Separating data driven and conceptually driven processes. Quarterly fournal of Experimental Psychology, 41A, 439-453.

Berry, D.C., \& Broadbent, D.E. (1988). Interactive tasks and the implicit-explicit distinction. British fournal of Psychology, 79, 251-272.

Berry, D.C., \& Dienes, Z. (1991). The relationship between implicit memory and implicit learning. British fournal of Psychology, 82, 359-373.

Bright, J.E.H., \& Burton, A.M. (1994). Past midnight: Semantic processing in an implicit learning task. Quarterly Journal of Experimental Psychology, 47A, 71-89.

Bright, J.E.H., \& Burton, A.M. (1998). Ringing the changes: Where abstraction occurs in implicit learning. European Fournal of Cognitive Psychology, 10, 113-130.

Brooks, L.R., \& Vokey, J.R. (1991). Abstract analogies and abstracted grammars: Comments on Reber (1989) and Mathews et al. (1989). Fournal of Experimental Psychology: General, 120, 316-323.

Churchill, E.F., \& Gilmore, D.J. (1998). Selection through rejection: Reconsidering the invariant learning paradigm. Quarterly Journal of Experimental Psychology, 51A, 1-17.

Cleeremans, A., Destrebecqz, A., \& Boyer, M. (1998). Implicit learning: News from the front. Trends in Cognitive Sciences, 2, 406-16.

Cock, J.J., Berry, D.C., \& Gaffan, E.A. (1994). New strings for old: The role of similarity processing in an incidental learning task. Quarterly Journal of Experimental Psychology, 47A, 1015-1034.

Dienes, Z., \& Altmann, G.T.M. (1997). Transfer of implicit knowledge across domains: How implicit and how abstract? In D.C. Berry (Ed.), How implicit is implicit learning? (pp. 107-123). Oxford, UK: Oxford University Press.

Dienes, Z., \& Berry, D. (1997). Implicit learning: Below the subjective threshold. Psychonomic Bulletin and Reviem, 4, $3-23$.

Gardiner, J.M., Dawson, A.J., \& Sutton, E.A. (1989). Specificity and generality of enhanced priming effects for selfgenerated study items. American fournal of Psychology, 102, 295-305.

Gomez, R.L. (1997). Transfer and complexity in artificial grammar learning. Cognitive Psychology, 33, 154-207.

Jacoby, L.L., \& Dallas, M. (1981). On the relationship between autobiographical memory and perceptual learning. Fournal of Experimental Psychology: General, 110, 306-340.

Jacoby, L.L., \& Hayman, C.A. (1987). Specific visual transfer in word identification. Fournal of Experimental Psychology: Learning, Memory, and Cognition, 13, 456-463.

Kolers, P.A. (1973). Remembering operations. Memory E Cognition, 1, 347-355.

Manza, L., \& Reber, A.S. (1997). Representing artificial grammars: Transfer across stimulus forms and modalities. In D.C. Berry (Ed.), How implicit is implicit learning? (pp. 73-106). Oxford, UK: Oxford University Press.

Mathews, R.C., Buss, R.R., Stanley, W.B., Blanchard Fields, F., Cho, J.R., \& Druhan, B. (1989). Role of implicit and explicit processes in learning from examples: A synergistic effect. Fournal of Experimental Psychology: Learning, Memory, and Cognition, 15, 1083-1100.

McGeorge, P., \& Burton, A.M. (1990). Semantic processing in an incidental learning task. Quarterly fournal of Experimental Psychology, 42A, 597-609.

Newell, B.R. (2000). An examination of the processes underlying implicit learning. Unpublished doctoral dissertation. University of New South Wales, Sydney, Australia.

Newell, B.R., \& Bright, J.E.H. (2001). The relationship between the structural mere exposure effect and the implicit learning process. Quarterly Fournal of Experimental Psychology, 54A, 1087-1104.

Newell, B.R., \& Bright, J.E.H. (2002). Well past midnight: Calling time on implicit invariant learning. European fournal of Cognitive Psychology, 14, 185-205. 
Reber, A.S. (1969). Transfer of syntactic structure in synthetic languages. Fournal of Experimental Psychology, 81, $115-119$.

Reber, A.S. (1989). Implicit learning and tacit knowledge. Fournal of Experimental Psychology: General, 118, 219-235.

Roediger, H.L., \& Blaxton, T.A. (1987). Effects of varying modality, surface features, and retention interval on priming in word-fragment completion. Memory $\mathbb{E}$ Cognition, 15, 379-388.

Schacter, D.L., Bowers, J., \& Booker, J. (1989). Intention, awareness, and implicit memory: The retrieval intentionality criterion. In S. Lewandowsky, J.C. Dunn, \& K. Kirsner (Eds.), Implicit memory: Theoretical issues (pp. 47-65). Hillsdale, NJ: Lawrence Erlbaum Associates, Inc.

Shanks, D.R., \& St. John, M.F. (1994). Characteristics of dissociable human learning systems. Behavioral and Brain Sciences, 17, 367-447.

Srinivas, K. (1993). Perceptual specificity in nonverbal priming. Fournal of Experimental Psychology: Learning, Memory and Cognition, 19, 582-602.

Stadler, M.A., Warren, J.L., \& Lesch, S.L. (2000). Is there cross-format transfer in implicit invariance learning? Quarterly Fournal of Experimental Psychology, 52A, 235-245.

Tulving, E., \& Schacter, D.L. (1990). Priming and human memory systems. Science, 247, 301-306.

Weldon, M.S. (1991). Mechanisms underlying priming on perceptual tests. Fournal of Experimental Psychology: Learning, Memory, and Cognition, 17, 526-541.

Whittlesea, B.W., \& Dorken, M.D. (1993). Incidentally, things in general are particularly determined: An episodicprocessing account of implicit learning. Fournal of Experimental Psychology: General, 122, 227-248.

Whittlesea, B.W.A., \& Wright, R.L. (1997). Implicit (and explicit) learning: Acting adaptively without knowing the consequences. Fournal of Experimental Psychology: Learning, Memory, and Cognition, 23, 181-200.

Willingham, D.B., Nissen, M.J., \& Bullemer, P. (1989). On the development of procedural knowledge. Fournal of Experimental Psychology: Learning, Memory, and Cognition, 15, 1047-1060.

Wright, R.L., \& Burton, A.M. (1995). Implicit learning of an invariant: Just say no. Quarterly Fournal of Experimental Psychology, 48A, 783-796. 


\section{APPENDIX}

Training strings used in Experiments 1, 2, and 4

$\begin{array}{lll}1328 & 8439 & 6213 \\ 4932 & 7153 & 2531 \\ 6539 & 2315 & 4392 \\ 8369 & 4536 & 8136 \\ 9873 & 9318 & 9538 \\ 1523 & 7341 & 3129 \\ 3246 & 9238 & 4839 \\ 5326 & 5398 & 6398 \\ 6753 & 4135 & 4673 \\ 3871 & 3416 & 3214\end{array}$

Test pairs used in Experiments 1 and 2

Test pairs used in Experiment 4

$\begin{array}{llll}3689 & 2579 & 5319 & 4785 \\ 2843 & 1247 & 2347 & 5962 \\ 8347 & 5891 & 3469 & 2815 \\ 6473 & 7154 & 4172 & 6215 \\ 1593 & 9542 & 5783 & 7612 \\ 3258 & 8216 & 7362 & 8721 \\ 5362 & 6149 & 9328 & 9841 \\ 7193 & 2715 & 7539 & 4192 \\ 8463 & 9654 & 4365 & 9278\end{array}$

$3422 \quad 7126 \quad 8423 \quad 5625$

$\begin{array}{llll}4724 & 2598 & 1536 & 7712\end{array}$

$\begin{array}{llll}8835 & 4859 & 9448 & 3714\end{array}$

$\begin{array}{llll}8113 & 9672 & 7742 & 6923\end{array}$

$5443 \quad 6175 \quad 6112 \quad 8496$

$\begin{array}{llll}2324 & 5264 & 4855 & 3261\end{array}$

$4739 \quad 4118 \quad 6224 \quad 3854$

$\begin{array}{llll}3146 & 6141 & 9289 & 4357\end{array}$

$\begin{array}{llll}9783 & 8944 & 5239 & 2669\end{array}$

Training strings used in Experiment 3

Test pairs used in Experiment 3

$\begin{array}{llll}9673 & 4253 & 7921 & 4378 \\ 2341 & 2346 & 8324 & 7982 \\ 3687 & 3917 & 4916 & 9243 \\ 8731 & 3218 & 9872 & 7365 \\ 3246 & 9316 & 8426 & 3461 \\ 4873 & 6783 & 3219 & 1926 \\ 8413 & 8613 & 8641 & 5463 \\ 5314 & 9341 & 3789 & 1972 \\ 3674 & 4392 & 3574 & 5724 \\ 1736 & 8356 & 4263 & 6754 \\ 8359 & 4328 & 6147 & 7683 \\ 3275 & 5793 & 3594 & 2945 \\ 2398 & 5312 & 1693 & 6251 \\ 7834 & 3216 & 5891 & 7923 \\ 1635 & 8263 & 6482 & 4398 \\ 4631 & 4831 & 5123 & 9182 \\ 2538 & 3981 & 4235 & 1862 \\ 9723 & 4361 & 9716 & 3128 \\ 7368 & 7436 & 7625 & 8352 \\ 3658 & 6532 & 2837 & 8264\end{array}$

